time for the metacarpophalangeal (MCP) joints might yield some disadvantages. Having the hand and arm immobilised for this long might discomfort the patients, thereby reducing acceptability, resulting in poor adherence. The longer imaging time might also increase the risk of motion-induced image degradation.

Objectives: The objective of this study was two-fold. Firstly, we investigated motion-induced image degradation of 2nd and 3rd MCP joints for two methods of standardised positioning of the hand. Secondly, the acceptability of HR-pQCT imaging was explored for patients with established Rheumatoid Arthritis (RA). Methods: Fifty patients with RA had their 2nd and 3rd MCP joints imaged by HR-pQCT. The patients were scanned two times, using a custom-made positioning splint, with and without an inflatable immobilisation device. In order to investigate acceptability, the patients were afterwards given a questionnaire regarding their procedure experience of HR-pQCT imaging with and without the inflatable hand immobilisation device. For each acquisition, the image quality was graded, and the number, width, depth and length of cortical interruptions were measured. Twenty percent of the acquisitions were reevaluated to determined intrareader reliability using the intraclass correlation coefficient (ICC).

Results: The acceptability regarding HR-pQCT imaging was high, with only $6 \%$ preferring conventional X-ray compared to $40 \%$ of the patients preferring HR-pQCT imaging. The remaining 54\% were indifferent to the modality. Seventy-four percent found it hard to keep their fingers at rest during the imaging. Fifty percent of the patients thought the inflatable hand immobilisation device helped keep their fingers at rest compared to only $6 \%$ who believed it impaired their ability to keep their fingers at rest. This was not observable in the image quality, however, as the overall image quality was high and no clinically relevant difference of the visual grading between the acquisitions with and without the inflatable hand immobilisation device was observed. The number, width, depth and length of cortical interruption all indicated excellent reproducibility as shown in table 1. No discernible difference between the two acquisitions was observed.

Table 1. Intraclass correlation coefficients for the number, width, depth and length of cortical interruptions, with and without the inflatable hand immobilization device.

\begin{tabular}{lll}
\hline & Acquisition 1 & Acquisition 2 \\
\hline Cortical interruptions number & $0.99(0.94$ to 1.00$)$ & $0.98(0.91$ to 1.00$)$ \\
Average cortical interruption width & $0.98(0.92$ to 0.99$)$ & $0.99(0.95$ to 1.00$)$ \\
Average cortical interruption depth & $0.98(0.92$ to 0.99$)$ & $0.97(0.89$ to 0.99$)$ \\
Average cortical interruption length & $0.93(0.75$ to 0.98$)$ & $0.98(0.94$ to 1.00$)$
\end{tabular}

Acquisition 1 - Without the inflatable hand immobilization device.

Acquisition 2 - With the inflatable hand immobilization device.

Data presented as mean ( $95 \%$ confidence intervals).

Conclusion: The high acceptability signifies the feasibility of the novel HR-pQCT imaging; this was evident by the fact that more patients preferred HR-pQCT imaging compared to conventional X-ray examination. The inflatable hand immobilisation device did not reduce motion-induced image degradation as the overall image quality was high for imaging of the MCP joints in both acquisitions. Our result, however, shows that the patients are more than capable of keeping their fingers at rest for the long acquisition time.

Disclosure of Interests: Rasmus Klose-Jensen: None declared, Kresten Krarup Keller: None declared, Bente Langdahl Grant/research support from: Amgen, NovoNordisk, Consultant of: Amgen Inc., Eli Lilly, UCB Pharma, Ellen Margrethe Hauge: None declared

DOI: 10.1136/annrheumdis-2020-eular.3728

\section{SAT0553 QUANTITATIVE ANALYSIS OF IMAGING FEATURES AT CHEST CT OF PULMONARY ARTERIAL AND VENOUS COMPONENTS IN SYSTEMIC SCLEROSIS- INTERSTITIAL LUNG DISEASE (SSC-ILD).}

C. Bruni ${ }^{1}$, M. Occhipinti ${ }^{2,3}$, G. Camiciottoli ${ }^{3}$, M. Bartolucci ${ }^{4}$, M. Pienn ${ }^{5}$, G. Lepri ${ }^{1}$,

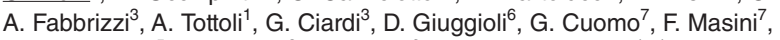
H. Olschewski ${ }^{5}$, F. Lavorini ${ }^{3}$, L. Calistri ${ }^{2}$, M. Matucci-Cerinic ${ }^{1} .{ }^{1}$ University of Florence, Rheumatology, Firenze, Italy; ${ }^{2}$ University of Florence, Radiology, Florence, Italy; ${ }^{3}$ University of Florence, Pulmonology, 50141, Italy; ${ }^{4}$ Careggi University Hospital, Radiology, Firenze, Italy; ${ }^{5}$ Ludwig Boltzmann Institute for Lung Vascular Research, Institute of Physiology, Graz, Austria; ${ }^{6}$ Policlinico of Modena University Hospital of Modena, Rheumatology, Modena, Italy; ${ }^{7}$ University of Campania Luigi Vanvitelli, Internal Medicine, Naples, Italy

Background: Interstitial lung disease (ILD) and pulmonary arterial hypertension carry a negative impact on SSc prognosis. Chest CT is the gold standard in assessing ILD and helps in evaluating associated vascular involvement.

Objectives: As qualitative analysis of CT scans is limited by low reproducibility and time constraints, we aimed at evaluating parenchymal and vascular features in SSc-ILD by quantitative analysis (QA) of CT scans and testing the relationship with clinical-functional data.
Methods: We prospectively enrolled 80 patients who underwent PFTs and chest CT scan spirometry gated at TLC on the same day. Clinical, lung functional and diffusion data, as well as disability indexes were collected. CT images were analyzed by a computational platform for texture analysis of ILD patterns (CALIPER), through Imbio LTA. It quantified the extent of normal pattern (NP \%), ground glass opacities (GG\%), reticulation (RET \%), honeycombing (HC \%), total ILD extent (ILD EXT \%) and hyperlucent (HL \%). Low density areas, representing emphysematous area, were also quantified (LDA \%). For lung vessel analysis, a software program developed by the Ludwig Boltzmann Institute for Lung Vascular Research was used. This software determined total, arterial, and venous vascular volumes (TV, AV, VV), and relative volumes (TV\%, AV\%, VV\%), as well as density and number for total, arterial and venous vessels.

Results: $43 / 80$ patients/CT scans were eligible for both software analyses, while $36 / 43$ for arterial and venous separation. TV\% and total vessel density were correlated positively with $\mathrm{mRSS}$ and negatively with \%FVC ( $\mathrm{r}=-0.537$ and $\mathrm{r}=-0.382)$ and \%TLC ( $\mathrm{r}=$ 0.511 and $r=-0.648$ ), while vessel tortuosity correlated positively with \%DLco. This was confirmed when separately analyzing arterial vessels, while VV\% negatively correlated with \%FVC, \%TLC and \%DLco. There was a positive correlation between \%ILD patterns and \%vascular volumes, being significant for TV\%-AV\%, total vessels and arterial density. Conversely, \%ILD patterns were negatively correlated with VV and number of veins detected, despite positive correlation between VV\% and ILD_EXT\%. When clustering patients according to \%FVC and \%DLco with $80 \%$ normal cutoff, \%FVC allowed clustering according to significantly different ILD patterns extents and vascular features, while \%DLCO for vascular features only. Moreover, the consecutive addition of functional impairment and worsening of ILD (from both normal \%FVC and \%Dlco, to \%DLco impairment only to both \%FVC and \%Dlco impairment), there was a significant increase in \%TV, \% AV and \%VV, with the exception of decrease in \%VV and venous density in patients with double impairment versus DLco single impairment.

Conclusion: This is the first study showing in SSc a direct correlation between ILD and the increase in lung vascular volume, which is characterized by increase in arterial volume and density and reduction in venous volume and number These results might be explained by the reduction of pulmonary volume due to fibrosis. However, also a para-physiological mechanism of redistribution of blood flow in lung areas, less involved by ILD, might be considered. Further studies on lung vessel quantification and distribution are ongoing.

Disclosure of Interests: Cosimo Bruni Speakers bureau: Actelion, Eli Lilly, Mariaelena Occhipinti Consultant of: Imbio, Gianna Camiciottoli: None declared, Maurizio Bartolucci: None declared, Michael Pienn: None declared, Gemma Lepri: None declared, Alessio Fabbrizzi: None declared, Alessandra Tottoli: None declared, Giuglia Ciardi: None declared, Dilia Giuggioli: None declared, Giovanna CUOMO: None declared, Francesco Masini: None declared, Horst Olschewski: None declared, Federico Lavorini: None declared, Linda Calistri: None declared, Marco Matucci-Cerinic Grant/research support from: Actelion, MSD, Bristol-Myers Squibb, Speakers bureau: Acetelion, Lilly, Boehringer Ingelheim DOI: 10.1136/annrheumdis-2020-eular.2177

\section{\begin{tabular}{|l|l}
\hline SAT0554 SONOGRAPHIC ASSESSMENT OF CALCIUM \\
\hline
\end{tabular} PYROPHOSPHATE DEPOSITION DISEASE AT WRIST. A FOCUS ON THE SCAPHO-LUNATE LIGAMENT.}

E. Cipolletta ${ }^{1}$, G. Smerilli ${ }^{1}$, R. Mashadi Mirza ${ }^{2}$, A. DI Matteo ${ }^{1,3}$, F. Salaffi $^{1}$, W. Grassi ${ }^{1}$, E. Filippucci ${ }^{1} .{ }^{1}$ Università Politecnica delle Marche, Rheumatology Unit, Jesi, Ancona, Italy; ${ }^{2}$ ULSS 6 Euganea, Dipartimento di Diagnostica per Immagini, Padova, Italy; ${ }^{3}$ University of Leeds, Leeds Institute of Rheumatic and Musculoskeletal Medicine, Leeds, United Kingdom

Background: Only few articles evaluated the wrist in calcium pyrophosphate deposition disease (CPPD), although it is the second most frequent target of CPPD. Very recently, in a computed tomography (CT) study ligamentous calcifications were reported as a highly specific feature of CPPD at wrist level (1).

Objectives: i) to determine the prevalence and distribution of the ultrasound (US) findings indicative of calcium pyrophosphate (CPP) crystal deposits at the wrist, with a particular focus on the dorsal aspect of the scapho-lunate ligament (SLL); ii) to investigate the diagnostic accuracy of US and conventional radiography (CR) in the evaluation of CPP crystal deposits at wrist level, iv) to assess the agreement between the different imaging techniques.

Methods: Consecutive patients with a "definite" diagnosis of CPPD according to the Ryan and McCarty criteria and disease controls were prospectively included in this cross-sectional single-centre study. Dorsal part of the SLL, triangular fibrocartilage complex (TFCC), and volar recess of the radio-lunate joint were explored using US (according to EULAR standard scans and OMERACT definitions), CR and CT.

Results: Sixty-one CPPD patients and 39 disease controls were enrolled. Two-hundred wrists were evaluated using both CR and US. CT data of 26 (13.0\%) wrists were available: 20 wrists in CPPD patients and 6 wrists in controls. CPP crystal deposits were found by US in at least one wrist in $95.1 \%$ of CPPD patients and in $15.4 \%$ of controls $(p<0.001)$. SLL calcification was reported in $83.6 \%$ of CPPD patients and in $5.1 \%$ of controls $(p<0.001)$. CPP crystal deposits were 
observed by US at the SLL and/or radio-lunate joint in $5.7 \%$ of wrists and $6.6 \%$ of CPPD patients, but not at the TFCC of the same wrist. On CR, calcifications were found in at least one wrist in $72.1 \%$ of CPPD patients and in $0 \%$ of controls $(p<0.001)$. Using the Ryan-McCarty criteria as a gold standard, the sensitivity, specificity and diagnostic accuracy were $0.72(0.59-0.83), 1.0(0.91-1.0)$ and 0.83 (0.74-0.90) for CR and 0.95 (0.86-0.99), 0.85 (0.69-0.94) and 0.91 (0.84-0.96) for US. Table 1 shows the agreement between the different imaging techniques.

Table 1. Agreement between US and the other imaging techniques in the evaluation of CPP crystal deposits at the wrist.

\begin{tabular}{lll}
\hline & US-CR $(n=200)$ & US-CT $(n=26)$ \\
\hline TFCC & $0.55(0.43-0.67)$ & $0.70(0.43-0.97)$ \\
SLL & $0.23(0.07-0.39)$ & $0.69(0.41-0.97)$ \\
RLJ & $0.25(0.09-0.41)$ & $0.46(0.12-0.80)$
\end{tabular}

Legend. $\mathbf{n}$ : number of the wrists, RLJ: volar recess of the radio-lunate joint. Values in brackets are the $95 \%$ confidence intervals of the Cohen's kappa.

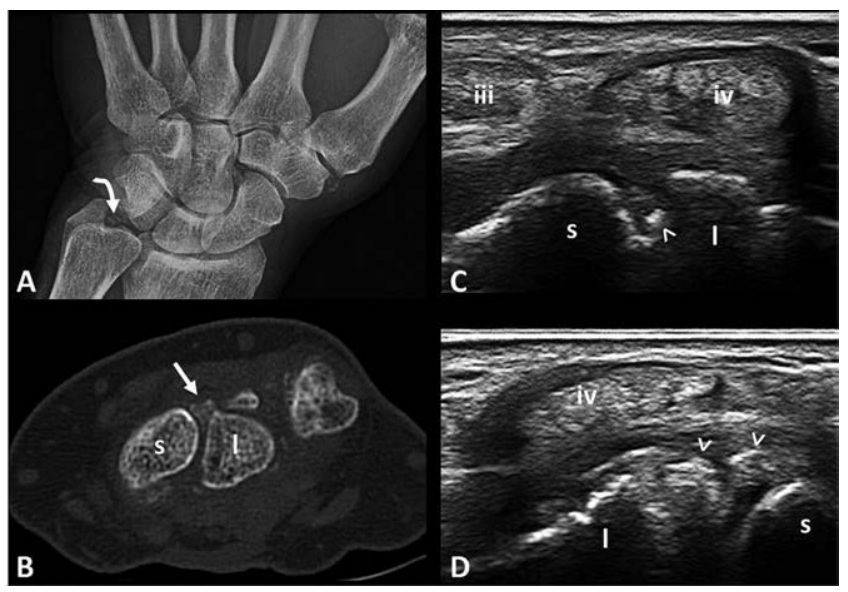

Figure 1 provides a pictorial evidence of the appearance of CPP crystal deposits in the SLL.A: CPP crystal deposits (curved arrow) at the TFCC. The SLL is not assessable due to superimposition of other bones.B: in the same patient of figure $1 \mathrm{~A}, \mathrm{CT}$ scan shows the presence of a calcification of the dorsal aspect of the SLL (arrow).C: dorsal longitudinal scan of the SLL: isolated hyperechoic spot (arrowheads) inside the ligament.D: dorsal longitudinal scan of the SLL showing the presence of a large aggregate extending towards the extensor tendons and hyperechoic spots (arrowheads) within it.Legend. iii: third extensor compartment, iv: fourth extensor compartment, I: lunate bone, s: scaphoid bone.

Conclusion: This study supports the diagnostic accuracy of US in evaluating wrist involvement in CPPD patients. SLL calcifications are a specific US finding of CPPD at wrist level.

References:

[1] Ziegeler K, Diekhoff T, Hermann S, et al. Low-dose computed tomography as diagnostic tool in calcium pyrophosphate deposition disease arthropathy: focus on ligamentous calcifications of the wrist. Clin Exp Rheumatol 2019;37:826-33

Disclosure of Interests: Edoardo Cipolletta: None declared, Gianluca Smerilli: None declared, Riccardo Mashadi Mirza: None declared, Andrea Di Matteo Grant/research support from: the publication was conducted while Dr. Di Matteo was an ARTICULUM fellow, Fausto Salaffi Speakers bureau: Dr. Salaffi reports personal fees from Bristol Myers Squibb, personal fees from Pfizer, personal fees from Novartis, personal fees from AbbVie, personal fees from Roche, personal fees from Merck Sharp \& Dohme Italia, outside the submitted work., Walter Grassi Speakers bureau: Prof. Grassi reports personal fees from AbbVie, personal fees from Celgene, personal fees from Grünenthal, personal fees from Pfizer, personal fees from Union Chimique Belge Pharma, outside the submitted work., Emilio Filippucci Speakers bureau: Dr. Filippucci reports personal fees from AbbVie, personal fees from Bristol-Myers Squibb, personal fees from Celgene, personal fees from Roche, personal fees from Union Chimique Belge Pharma, personal fees from Pfizer, outside the submitted work.

DOI: 10.1136/annrheumdis-2020-eular.720

\section{SAT0555 MUSCULOSKELETAL ULTRASOUND IN MONITORING RESPONSE TO JAKI IN RHEUMATOID ARTHRITIS PATIENTS: RESULTS FROM A LONGITUDINAL STUDY}

E. Cipriano ${ }^{1}$, F. Ceccarelli ${ }^{1}$, F. R. Spinelli ${ }^{1}$, C. Garufi ${ }^{1}$, I. Duca ${ }^{1}$, S. Mancuso ${ }^{1}$, C. Alessandri ${ }^{1}$, M. DI Franco ${ }^{1}$, R. Priori ${ }^{1}$, V. Riccieri ${ }^{1}$, R. Scrivo ${ }^{1}$, C. Perricone ${ }^{2}$,
G. Valesini ${ }^{1}$, F. Conti ${ }^{1}{ }^{1}$ Sapienza University of Rome, Rome, Italy; ${ }^{2}$ Università di Perugia, Perugia, Italy

Background: Therapeutic approach of rheumatoid arthritis (RA) patients has been enriched by the introduction of small molecules. In particular Jak inhibitors (JAKi), baricitinib and tofacitinib, demonstrated their efficacy in patients naïve or resistant to biological treatments in randomized controlled trials. Moreover, these drugs seem to be able to prevent radiographic progression. To date few data are available from the real life context. Ultrasonographic (US) assessment has became a valid imaging tool in the management of RA patients in clinical practice, allowing the evaluation of joint inflammatory status. Together with clinimetric assessment, US could provide a comprehensive assessment of drug response. Objectives: In the present study we aimed at assessing the early response to JAKi treatment by using musculoskeletal US.

Methods: In this prospective longitudinal study, we collected data about all consecutive active RA patients starting treatment with JAKi. RA was diagnosed according to the 2010 ACR/EULAR criteria. At each visit, clinical and laboratory data were collected in a standardized and computerized form, including demographics, past medical history, co-morbidities, previous and concomitant treatments. According with study protocol, all patients underwent clinical and US assessment at the following time-points: baseline (T0), 4 weeks (T1) and 12 weeks (T2). Clinical evaluation included tender and swollen joint counts (0-28) patients global health assessment. C-reactive protein (CRP) levels were registered and disease activity was calculated by disease activity score (DAS) in 28 joints by using CRP (DAS28-CRP). A systematic multiplanar grey-scale and power Doppler $(\mathrm{pD})$ US examination was performed by using MyLab Eight Exp Machine (Esaote, Florence, Italy) at level of 22 joints (bilateral I-V metacarpophalangeal, I-V proximal interphalangeal, wrist). According with OMERACT definitions (1) we assessed the presence of synovial effusion, hypertrophy and $\mathrm{pD}$, that were scored according to a semi-quantitative scale (0-3). A total US inflammatory score (0-198) was obtained by their sum.

Results: We enrolled 91 patients [F/M 77/14; median age 60.0 years (IQR 15.5) median disease duration 144 months (IQR 126)]. Of these patients, 54 (59.3\%) were treated by baricitinib and the remaining 37 by tofacitinib. At baseline we found a median US inflammatory score of 20 (IQR 18.7) and a median DAS28CRP of 5.0 (IQR 1.56). US assessment demonstrated significant reduction in the median values of inflammatory score already at T1 [median 13 (IQR 14.7), $\mathrm{p}<0.0001$ ], that was maintained at T2 [median 10 (IQR 11), $\mathrm{p}<0.0001$ ]. These results are represented in figure 1 . Similar to US inflammatory score, a significant reduction was registered for DAS28-CRP median values [T1 3.5 (IQR 1.73) $\mathrm{p}<0.0001$; T2 3.3 (IQR 1.8), $\mathrm{p}<0.0001$ ]. No significant differences were found when subgrouping patients according with different JAKi drug, in terms US and clinimetric assessment

Conclusion: In the present study, specifically designed to evaluate the US-detected efficacy of JAKi in RA patients, we demonstrated in a real life setting a significant, early and sustained improvement of inflammatory joint status.

Figure 1. Box and whiskers plot (median, quartiles, range) of US inflammatory score of $91 \mathrm{RA}$ patients at baseline (T0), after 4 weeks (T1) and 12 weeks (T2) of treatment with JAKi. P values were referred to the comparison with baseline.

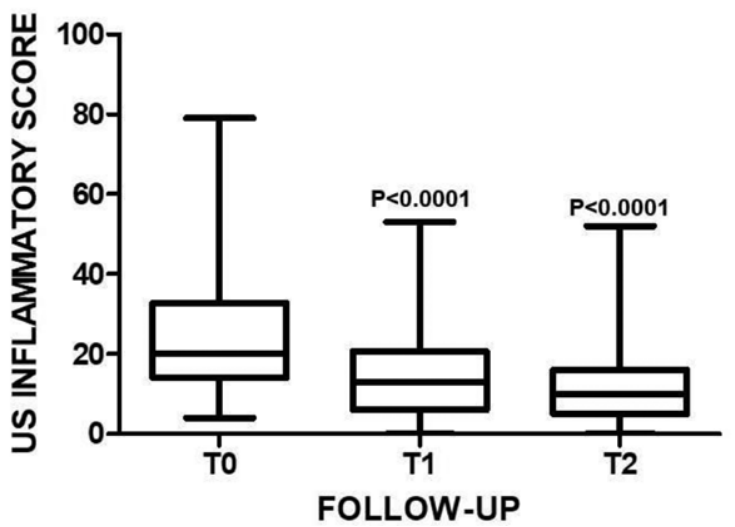

References:

[1] Wakefield et al, J Rheumatol 2005

Disclosure of Interests: enrica cipriano: None declared, Fulvia Ceccarelli: None declared, Francesca Romana Spinelli Grant/research support from: Pfizer, Consultant of: Novartis, Gilead, Lilly, Sanofi, Celgene, Speakers bureau: Lilly, Cristina Garufi: None declared, Ilaria Duca: None declared, Silvia Mancuso: None declared, cristiano alessandri Grant/research support from: Pfizer, Manuela Di Franco: None declared, Roberta Priori: None declared, Valeria Riccieri: None 